\title{
THE SPECTRUM OF AN OPERATOR ON AN INTERPOLATION SPACE
}

BY

JAMES D. STAFNEY

1. Introduction. There are two related problems solved in this paper. The first problem is to describe the structure space of a Banach algebra obtained from two given commutative Banach algebras by analytic interpolation. The solution to this problem is given in Theorem 5.5, which is really the main theorem of the paper. The second problem is to determine the spectrum of a bounded operator on a Banach space that is obtained from two given Banach spaces by analytic interpolation. One observes that the spectrum of the operator is the structure space of a related commutative Banach algebra, and then the previous result can be used. The main theorems for the result on the spectrum of an operator are 6.7 and 6.10. It turns out that the main theorem in $\$ 6$, Theorem 6.7 , gives an upper bound, a containing set, for the spectrum of the operator on the interpolation space in terms of the spectra of the operator on the given two spaces. This upper bound is the best possible one that depends only on the given two spectra. Theorem 6.10 shows that the upper bound can be improved if more is known about the operator. In $\$ 7$ we give an example, 7.3, to show that the bound in Theorem 6.7 need not be attained. The example also shows that Theorem 6.10 is stronger than Theorem 6.7.

$\S \S 2,3$ and 4 are mainly lemmas and definitions needed in the later sections; Theorem 2.7, which is a generalization of the three lines theorem due to Calderón, will be an important tool. A similar-type generalization of the three lines theorem is given in Lemma 5.3, which may also be of independent interest. In $\$ 2$ we give the definition of analytic interpolation, which was first introduced by A. P. Calderón at the conference on functional analysis in the memory of S. Banach, in Warsaw, 1960; we also state several of the relevant properties of analytic interpolation. In $\$ 3$ we introduce the notion of a RIS, which is essentially the way in which two commutative Banach algebras must be related so that their structure spaces interpolate in the natural manner. We also obtain some basic properties of a RIS which are needed later. In order to obtain our main theorem it seems to be necessary to compare analytic interpolation with what we call direct interpolation, which we define in $\$ 4$. The theorem of $\$ 4$ contains results which are of use in $\$ 5$. This theorem would be elementary except for the fact that the proof requires Calderón's generalized three lines theorem mentioned above.

Presented to the Society, January 24, 1968; received by the editors November 21, 1967 and, in revised form, February 3, 1969. 
Evidently, the results of this paper apply only in those situations where the spaces involved are related by analytic interpolation. There are several papers which are concerned with the problem of realizing a parametrized family of Banach spaces as a family of spaces related by analytic interpolation. In particular, Calderón gives some rather general results in this direction in $\$ \$ 13$ and 14 of [2]. We also point out that results in [4], [5] and [6] are related to results in this paper since the $l_{p}$ spaces are related by analytic interpolation.

2. The method of analytic interpolation. For the convenience of the reader we use this section to state the definition of analytic interpolation precisely as it appears in [2, p. 114] and to state several results appearing in [2]. Only Lemma 2.5 is new.

2.1. An interpolation pair $\left(B^{0}, B^{1}\right)$ is a pair of complex Banach spaces $B^{0}, B^{1}$, continuously embedded in a complex topological vector space $V$. If $x$ is an element of $B^{i}, i=0,1$, we denote its norm by $\|x\|_{i}$ or $\|x\|_{B^{i}}$. If in $B^{0} \cap B^{1}$ we introduce the norm $\|x\|_{B^{0} \cap B^{1}}=\max \left(\|x\|_{0},\|x\|_{1}\right)$, then $B^{0} \cap B^{1}$ becomes a Banach space.

Similarly, if we consider the space $B^{0}+B^{1}$ and introduce in it the norm $\|x\|_{B^{0}+B^{1}}$ $=\inf \left[\|y\|_{0}+\|z\|_{1}\right]$, where the infimum is taken over all pairs $y, z, y \in B^{0}, z \in B^{1}$, such that $y+z=x$, then $B^{0}+B^{1}$ also becomes a Banach space.

Since $B^{0}$ and $B^{1}$ are continuously embedded in $V$, it is evident that $B^{0} \cap B^{1}$ and $B^{0}+B^{1}$ are also continuously embedded in $V$.

Given an interpolation pair we consider functions $f(\xi), \xi=s+i t$ defined in the strip $0 \leqq s \leqq 1$ of the $\xi$-plane, with values in $B^{0}+B^{1}$ continuous and bounded with respect to the norm of $B^{0}+B^{1}$ in $0 \leqq s \leqq 1$ and analytic in $0<s<1$, and such that $f(i t) \in B^{0}$ is $B^{0}$-continuous and tends to zero as $|t| \rightarrow \infty, f(1+i t) \in B^{1}$ is $B^{1}$ continuous and tends to zero as $|t| \rightarrow \infty$. In this linear space of functions which we denote by $\mathscr{F}\left(B^{0}, B^{1}\right)$ we introduce the norm

$$
\|f\|_{\mathscr{F}}=\max \left[\sup _{t}\|f(i t)\|_{0}, \sup _{t}\|f(1+i t)\|_{1}\right] .
$$

Then $\mathscr{F}$ becomes a Banach space.

Given a real number $s, 0 \leqq s \leqq 1$, we consider the subspace $B_{s}=\left[B^{0}, B^{1}\right]_{s}$ of $B^{0}+B^{1}$ defined by $B_{s}=\left\{x \mid x=f(s), f \in \mathscr{F}\left(B^{0}, B^{1}\right)\right\}$ and introduce in it the norm $\|x\|_{s}=\|x\|_{B_{s}}=\inf \|f\|_{\mathscr{F}}, f(s)=x$. We say that the space $B_{s}$ with norm \|\|$_{s}$ is obtained by the method of analytic interpolation.

Throughout the paper $D$ will denote the complex number $\xi$ with $0 \leqq \operatorname{Re}(\xi) \leqq 1$. Let $\mathscr{G}$ denote the subspace of $\mathscr{F}$ consisting of elements of the form $f(\xi)=\sum c_{n}(\xi) x_{n}$ $(n=1,2, \ldots, N)$ where $c_{n}(\xi)$ is continuous on $D$, vanishes at infinity and is analytic on the interior of $D$ and where each $x_{n}$ is in $B^{0} \cap B^{1}$.

For the convenience of the reader we state three lemmas from [2]. These lemmas appear in $\$ \$ .2,9.3$ and 10.5 , respectively.

2.2 Lemma. $\mathscr{G}$ is dense in $\mathscr{F}$. 
2.3 Lemma. $B^{0} \cap B^{1}$ is dense in $B_{s}, 0<s<1$.

2.4 Lemma. Let $(A, B)$ be an interpolation pair and suppose that $A$ and $B$ are Banach algebras with the property that multiplications in $A$ and $B$ coincide in $A \cap B$. Then $A \cap B$ is a subalgebra of both $A$ and $B$ and for each $s, 0<s<1$, and for $x, y$ in $A \cap B$ we have

$$
\|x y\|_{C} \leqq\|x\|_{C}\|y\|_{C}, \quad C=[A, B]_{s},
$$

so that multiplication can be extended continuously to $C$, which then becomes a Banach algebra.

2.5 Lemma. For each $x$ in $B^{0} \cap B^{1}$ and $0<s<1$,

$$
\|x\|_{s}=\inf \left\{\|f\|_{\mathscr{F}}: f(s)=x, f \in \mathscr{G}\right\} .
$$

Proof. Let $x$ be in $B^{0} \cap B^{1}$ and $\varepsilon$ an arbitrary positive real number. The function $e^{(\xi-s)^{2}} x=f(\xi)$ is in $\mathscr{G}$ and $f(s)=x$. It is clear from the definition of the norm \|\|$_{s}$ that, for some $g$ in the subspace $\mathscr{N}$ of $\mathscr{F}$, consisting of functions with value 0 at $s$,

$$
\|f-g\|_{\mathscr{F}}<\|x\|_{s}+\varepsilon .
$$

We need the following proposition.

Proposition. $\mathscr{G} \cap \mathscr{N}$ is dense in $\mathscr{N}$ with respect to the norm of $\mathscr{F}$ restricted to $\mathscr{N}$.

We will first give a proof of the proposition and then continue with the proof of the lemma. Let $g$ be in $\mathcal{N}$ and let $r$ denote a conformal map of the interior of $D$ onto the circle $|z|<1$ such that $r(s)=0$. It is clear that $r$ has a continuous extension to the boundary of $D$ so that the modulus on the boundary is 1 . Let $r$ denote the extended function. Since $g(s)=0$ it is clear that $g / r$ is in $\mathscr{F}$. By Lemma 2.2 there is a $g_{1}$ in $\mathscr{G}$ such that $\left\|g_{1}-g / r\right\|_{\mathscr{F}}<\varepsilon$. Since the modulus of $r$ does not exceed 1 , $\left\|r g_{1}-g\right\|_{\mathscr{F}}<\varepsilon$. Since $r(s)=0, r g_{1}$ is in $\mathscr{G} \cap \mathscr{N}$. This proves the proposition.

Continuing with the proof of Lemma 2.5, we choose $g_{1}$ in $\mathscr{G} \cap \mathscr{N}$ so that $\left\|g-g_{1}\right\|_{\mathscr{F}}<\varepsilon$. Since $f-g_{1} \in \mathscr{G},\left(f-g_{1}\right)(s)=x$ and $\left\|f-g_{1}\right\|_{\mathscr{F}}<\|x\|_{s}+2 \varepsilon$, the proof of the lemma is complete.

The following lemma is only a slight variation of $[2, \S 4]$.

2.6 Lemma. If $T$ is a linear transformation on $B^{0} \cap B^{1}$ with operator norm $M_{k}$ with respect to the norm \|\|$_{k}$ restricted to $B^{0} \cap B^{1}$, then the operator norm of $T$ with respect to \|\|$_{s}$ does not exceed $M_{0}^{1-s} M_{1}^{s}$.

Proof. We have assumed that $B^{0} \cap B^{1}$ is dense in both $B^{0}$ and $B^{1}$. Let $T_{0}$ and $T_{1}$ denote the unique bounded linear extensions of $T$ to $B^{0}$ and $B^{1}$, respectively. Define an extension $U$ of $T$ to $B^{0}+B^{1}$ by $U(x+y)=T_{0} x+T_{1} y, x \in B^{0}, y \in B^{1}$. One easily shows that $U$ is a well-defined extension of $T$ satisfying the conditions of $[2, \S 4]$; thus, our conclusion follows from the latter.

The following theorem and its corollary are special cases of (ii) in [2, \$9.4]. 
2.7 THEOREM. For each real number $s, 0<s<1$, and each $f$ in $\mathscr{F}$,

$$
\|f(s)\|_{s} \leqq \sup _{y}\|f(i y)\|_{0}^{1-s} \sup _{y}\|f(1+i y)\|_{1}^{s}
$$

where the supremum in each case is taken over all real values of $y$.

2.8 CoRollary. For each $x$ in $B^{0} \cap B^{1}$ we have $\|x\|_{s} \leqq\|x\|_{0}^{1-s}\|x\|_{1}^{s}, 0<s<1$.

Proof. Let $f_{\varepsilon}(w)=\left(e^{\varepsilon w^{2}}\right) x$, apply the previous theorem and let $\varepsilon$ tend to 0 through positive real values.

3. Definition and properties of a RIS. The purpose of this section is to introduce the notion of a RIS (see 3.1) and to obtain some of the properties (3.2-3.5) which will be needed in the rest of the paper. We begin, however, by recalling some facts about commutative Banach algebras.

Let $(A,||)$ be a commutative Banach algebra with identity. From now on in this paper the assumption of being a commutative Banach algebra will include having an identity. We will let sp ( $a, A)$ denote the spectrum of an element $a$ in $A$, i.e., the set of complex numbers $\lambda$ such that $\lambda e-a$ does not have an inverse in $A$ (where $e$ is the identity in $A$ ). Let $r(a)$ denote the spectral radius, i.e., the radius of the smallest circle with center 0 which contains sp $(a, A)$. Also, for a set $E$ in the complex plane, we let $R(E)$ denote the rational functions with poles in the complement of $E$. In case sp $(a, A) \subset E, R_{a}(E)$ will denote the algebra of elements $f(a)$ in $A$ where $f$ is in $R(E)$ and $f(a)$ is defined algebraically. In general, if $f$ is an analytic function on a neighborhood of $\operatorname{sp}(a, A)$, we let $f(a)$ denote the element of $A$ defined in the usual manner by a vector valued complex integral (see [7, Chapter V]). Finally, by the structure space of $A$ we mean the set of all nonzero multiplicative linear functionals $\phi$ on $A$. The fact that the structure space has a natural topology will not be used in this paper. We recall the following well-known facts.

(3.A.1) $\operatorname{sp~}(a, A)=\{\phi(a): \phi$ in the structure space of $A\}$.

(3.A.2) For each $a$ in $A, \lim _{n}\left|a^{n}\right|^{1 / n}=r(a)$.

(3.A.3) $r(a) \leqq|a|, a \in A$.

(3.A.4) If $f$ is analytic on a neighborhood of $\operatorname{sp}(a, A)$, then $\operatorname{sp}(f(a), A)=$ $f(\operatorname{sp}(a, A))$. in $A$.

(3.A.5) $\operatorname{sp}(a, A)=\operatorname{sp}\left(a, R_{a}(\operatorname{sp}(a, A))^{-}\right)$where $R_{a}(E)^{-}$is the closure of $R_{a}(E)$

(3.A.6) If $U$ is an open set which contains $\operatorname{sp}(a, A)$, there is a constant $c$ such that $|f(a)| \leqq c \sup _{z \in U}|f(z)|, f$ analytic on $U$.

3.1 Definition. Let $\left(A_{0},||_{0}\right)$ and $\left(A_{1},||_{1}\right)$ be commutative Banach algebras which are continuously embedded in a topological vector space $W$. Suppose that there is an element $x$ in $A_{0} \cap A_{1}$ such that the sets $E_{k}=\operatorname{sp}\left(x, A_{k}\right), k=0,1$, satisfy the following conditions:

(3.1.1) $E_{0}$ is a subset of the interior of $E_{1}$; 
(3.1.2) for each $f$ in $R\left(E_{1}\right), f(x)$ is well defined, i.e., the same element of $W$ is obtained when $f(x)$ is computed in $A_{0}$ and in $A_{1}$;

(3.1.3) in $A_{1}$, the set $\left\{f(x): f \in R\left(E_{1}\right)\right\}$ is dense.

When these conditions are met, we say that $\left(A_{0}, A_{1}, W, x\right)$ is a rational interpolation system (RIS).

In the remainder of this section, the notation will be that of 3.1. In particular, $\left(A_{0}, A_{1}\right)$ is an interpolation pair. We will let $A_{s}=\left[A_{0}, A_{1}\right]_{s}, 0<s<1$, and will let $M_{s}$ denote the norm of $A_{s}$.

3.2 LemMA. $A_{1} \subset A_{0}$ and there is a constant $c$ such that

$$
|y|_{0} \leqq c|y|_{1}, \quad y \in A_{1}
$$

and

$$
M_{s}(y) \leqq c|y|_{1}, \quad y \in A_{1} .
$$

Also, $R_{x}\left(E_{1}\right)$ is dense in $A_{s}$ with norm $M_{s}$.

Proof. We first note that since $E_{0}$ is contained in the interior of $E_{1}$, it follows from (3.A.6) that for some constant $c$,

$$
|f(x)|_{0} \leqq c \sup _{z \in E_{1}}|f(z)| \leqq c|f(x)|_{1}, \quad f \in R\left(E_{1}\right) .
$$

Let $y \in A_{1}$. Since $R_{x}\left(E_{1}\right)$ is dense in $A_{1}$, we can choose a sequence $f_{n}$ in $R\left(E_{1}\right)$ such that $\lim \left|f_{n}(x)-y\right|_{1}=0$. By (3.2.3)

$$
\left|f_{n}(x)-f_{m}(x)\right|_{0} \leqq c\left|f_{n}(x)-f_{m}(x)\right|_{1},
$$

for all $m, n$, so there is a $y_{1}$ in $A_{0}$ such that $\left|f_{n}(x)-y_{1}\right|_{0}$ converges to 0 . Since $f_{n}(x)$ converges to $y$ and $y_{1}$ in the topology of $W, y=y_{1}$; thus, $y \in A_{0}$ and $|y|_{0} \leqq c|y|_{1}$. This proves that $A_{1} \subset A_{0}$ and (3.2.1). To prove (3.2.2) we consider functions $q_{\varepsilon}(\xi)=e^{\varepsilon(\xi)^{2}} y$ for each positive $\varepsilon$. Evidently, $q_{\varepsilon} \in \mathscr{F}\left(A_{0}, A_{1}\right)$ and

$$
M_{s}(y) \leqq \lim _{\varepsilon \rightarrow 0}\left\|q_{\varepsilon}\right\|_{\mathscr{F}_{\left(A_{0}, A_{1}\right)} \leqq c|y|_{1} .}
$$

To prove the last assertion we note that by Lemma $2.3, A_{0} \cap A_{1}$ is dense in $A_{s}$ with respect to the norm $M_{s}$. From what we have already shown, $A_{1}=A_{0} \cap A_{1}$ and there is a constant $c$ such that $M_{s} \leqq c|\quad|{ }_{1}$ on $A_{1}$. Since by assumption $R_{x}\left(E_{1}\right)$ is dense in $A_{1}$ with respect to the norm | $\left.\right|_{1}, R_{x}\left(E_{1}\right)$ must be dense in $A_{1}$ with respect to the norm $M_{s}$. This completes the proof.

3.3 Lemma. The multiplication on $R_{x}\left(E_{1}\right)$ has a unique extension to $A_{s}$ making $\left(A_{s}, M_{s}\right)$ a commutative Banach algebra.

Proof. By 3.2, $R_{x}\left(E_{1}\right)$ is dense in $A_{s}$, and $R_{x}\left(E_{1}\right)$ is clearly a commutative algebra. Therefore, if the multiplication on $R_{x}\left(E_{1}\right)$ has an extension making $\left(A_{s}, M_{s}\right)$ a Banach algebra, then the extended multiplication must be commutative and unique. The fact that there is an extension of the multiplication of the desired type follows 
from Lemma 2.4 if we can show that the multiplications agree on $A_{0} \cap A_{1}$ and not just on $R_{x}\left(E_{1}\right)$. Let $a, b$ be in $A_{0} \cap A_{1}$ and let $a b$ and $a \odot b$ denote the product in the algebras $A_{1}$ and $A_{0}$, respectively. Since $A_{1} \subset A_{0}$, both $a b$ and $a \odot b$ are in $A_{0}$. Since $R_{x}\left(E_{1}\right)$ is dense in $A_{1}$ we can choose $a_{1}, b_{1}$ in $R_{x}\left(E_{1}\right)$ so that $\left|a-a_{1}\right|_{1}<\varepsilon$ and $\left|b-b_{1}\right|_{1}<\varepsilon$ where $\varepsilon$ is some positive real number. By assumption, $a_{1} b_{1}=a_{1} \odot b_{1}$. The following inequalities are now clear.

$$
\begin{aligned}
|a b-a \odot b|_{0} & =\left|a b-a_{1} b_{1}+a_{1} \odot b_{1}-a \odot b\right|_{0} \\
& \leqq c\left|a b-a_{1} b_{1}\right|_{1}+\left|a_{1} \odot b_{1}-a \odot b\right|_{0} \\
& \leqq c\left(|a|_{1} \varepsilon+\left|b_{1}\right|_{1} \varepsilon\right)+|a|_{0} c \varepsilon+\left|b_{1}\right|_{0} c \varepsilon .
\end{aligned}
$$

Since $\varepsilon$ is arbitrary, we see that $a b=a \odot b$, so the multiplications agree on $A_{0} \cap A_{1}$. This completes the proof.

We let $H_{s}$ denote the structure space of $A_{s}$.

3.4 LEMMA. If $z$ is an element in $E_{1}$ such that

$$
|f(z)| \leqq M_{s}(f(x)), \quad f \in R\left(E_{1}\right),
$$

then the condition $\phi(f(x))=f(z), f \in R\left(E_{1}\right)$ well defines $\phi$ as a function on $R_{x}\left(E_{1}\right)$, and $\phi$ has a unique extension in $H_{s}$.

Proof. Let $f, g$ be elements of $R\left(E_{1}\right)$ such that $f(x)=g(x)$ in $A_{0}$ and $A_{1}$. Since $f-g$ is analytic on $E_{1}$, it follows from the spectral mapping theorem that

$$
\operatorname{sp}\left((f-g)(x), A_{k}\right)=(f-g)\left(\operatorname{sp}\left(x, A_{k}\right)\right), \quad k=0,1 .
$$

Therefore, since $\operatorname{sp}\left(0, A_{k}\right)=\{0\}, f-g \equiv 0$ on $E_{1}$. This shows that $\phi$ is well defined on $R_{x}\left(E_{1}\right)$. If we let $f$ be the rational function which is identically 1 , then since $\phi(f(x))=f(z)=1$ we see that $\phi$ is not identically 0 . From this, the definition of $\phi$ and (3.4.1), we conclude that $\phi$ is a nonzero multiplicative linear functional on $R_{x}\left(E_{1}\right)$ which is continuous with respect to the norm $M_{s}$. From the last assertion in 3.2 we know that $R_{x}\left(E_{1}\right)$ is dense in $A_{s}$ with norm $M_{s}$, so we conclude that $\phi$ has a unique extension in $H_{s}$. This completes the proof.

3.5 Lemma. For each $z$ in $E_{1}$ there is a unique $\lambda(z, \cdot)$ in the structure space of $A_{1}$ such that $\lambda(z, f(x))=f(z), f \in R\left(E_{1}\right)$. Furthermore, for each $y$ in $A_{1}$ the map $z \rightarrow \lambda(z, y)$ is continuous on $E_{1}$ and analytic on the interior of $E_{1}$. Also, for each $y$ in $A_{1}$ and $z$ in $E_{0},|\lambda(z, y)| \leqq|y|_{0}$.

Proof. For each $z$ in $E_{1}$ we define $\lambda_{1}(z, \cdot)$ by the condition $\lambda_{1}(z, f(x))=f(z)$, $f \in R\left(E_{1}\right)$. By the same argument that is used in the proof of 3.4, we conclude that $\lambda_{1}(z, \cdot)$ is a well-defined nonzero multiplicative linear functional on $R_{x}\left(E_{1}\right)$. Since $R_{x}\left(E_{1}\right)$ is dense in $A_{1}$ and

$$
|f(z)| \leqq|f(x)|_{1}, \quad f \in R\left(E_{1}\right),
$$

$\lambda_{1}(z, \cdot)$ has a unique extension $\lambda(z, \cdot)$ in the structure space of $A_{1}$. Now let $y$ be an 
element in $A_{1}$. There is a sequence $f_{n}$ in $R_{x}\left(E_{1}\right)$ such that $\left|f_{n}(x)-y\right|_{1}$ converges to 0 . Therefore, for each value of $z$ in $E_{1}$,

$$
\lambda(z, y)=\lim _{n} \lambda\left(z, f_{n}(x)\right) .
$$

Since each linear functional $\lambda(z, \cdot)$ has norm 1 , we have

$$
\left|f_{n}(z)-f_{m}(z)\right| \leqq\left|f_{n}(x)-f_{n}(x)\right|, \quad z \in E_{1} ; \quad m, n=1,2, \ldots
$$

From the above observations, we see that $\lambda(z, y)$ is the uniform limit of the functions $f_{n}(z)$ on $E_{1}$. This proves the second assertion of the lemma.

If $z \in E_{0}$, then $|f(z)| \leqq|f(x)|_{0}, f \in R\left(E_{1}\right)$. Since $f(z)=\lambda(z, f(x))$ we have that

$$
|\lambda(z, f(x))| \leqq|f(x)|_{0}, \quad f \in R\left(E_{1}\right) .
$$

If $y \in A_{1}$, then there is a sequence $f_{n}$ in $R\left(E_{1}\right)$ such that $\left|f_{n}(x)-y\right|_{1}$ converges to 0 . By $3.2\left|f_{n}(x)\right|_{0}$ converges to $|y|_{0}$. Since $\lambda(z, \cdot)$ is continuous on $A_{1}, \lambda\left(z, f_{n}(x)\right)$ converges to $\lambda(z, y)$ as $n \rightarrow \infty$. This shows that $|\lambda(z, y)| \leqq|y|_{0}$, which completes the proof of the lemma.

4. Direct interpolation. In order to prove our main theorem in the next section, it is necessary to consider what we will call direct interpolation. This interpolation formula is not new; it appears, for example, in [1, p. 470]. In the process of proving our main theorem, we will make some comparisons between analytic interpolation and direct interpolation; these comparisons are interesting in themselves. To avoid further notation, we will define direct interpolation for our special situation.

Let $\left(A_{0}, A_{1}, W, x\right)$ be a RIS as defined in (3.1). For each real number $s, 0<s<1$, we define the norm $N_{s}$ or $N$ on $A_{1}=A_{0} \cap A_{1}$ as follows: for each $a$ in $A_{1}$

$$
N(a)=\inf \sum_{j}\left|a_{j}\right|_{0}^{1-s}\left|a_{j}\right|_{1}^{s}
$$

where the inf is taken over all finite sums $a=\sum_{j} a_{i}, a_{j} \in A_{1}$. As we shall see in the next proposition, $A_{1}$ becomes a normed algebra with the norm $N$. We will let $K_{s}$ or $K$ denote the structure space of this algebra.

4.1. THEOREM.

(4.1.1) $N$ is an algebra norm on $A_{1}$ and $N(1)=1$.

(4.1.2) $N$ is the unique maximal norm on $A_{1}$ such that $N(a) \leqq|a|_{0}^{1-s}|a|_{1}^{s}, a \in A_{1}$.

(4.1.3) $M(a) \leqq N(a), a \in A_{1}$.

(4.1.4) If $z$ is an element in $E_{1}$ such that $|f(z)| \leqq N_{s}(f(x)), f \in R\left(E_{1}\right)$, then the condition $\phi(f(x))=f(z), f \in R\left(E_{1}\right)$ well defines $\phi$ as a function on $R_{x}\left(E_{1}\right)$, and $\phi$ has a unique extension in $K_{s}$.

Proof. To prove (4.1.1) let $a$ and $b$ be in $A_{1}$ and suppose that $\sum a_{j}$ and $\sum b_{k}$ are finite sums which are equal to $a$ and $b$, respectively. Suppose that

$$
N(a)+\varepsilon \geqq \sum\left|a_{j}\right|_{0}^{1-s}\left|a_{j}\right|_{1}^{s},
$$


and similarly for $b$, where $\varepsilon$ is an arbitrary positive real number. Then,

$$
N(f+g) \leqq \sum\left|a_{j}\right|_{0}^{1-s}\left|a_{j}\right|_{1}^{s}+\sum\left|b_{k}\right|_{0}^{1-s}\left|b_{k}\right|_{1}^{s} \leqq 2 \varepsilon+N(a)+N(b)
$$

and

$$
\begin{aligned}
N(a b) & \leqq \sum\left|a_{j} b_{k}\right|_{0}^{1-s}\left|a_{j} b_{k}\right|_{1}^{s} \\
& \leqq \sum\left(\left|a_{j}\right|_{0}\left|b_{k}\right|_{0}\right)^{1-s}\left(\left|a_{j}\right|_{1}\left|b_{k}\right|_{1}\right)^{s} \leqq(N(a)+\varepsilon)(N(b)+\varepsilon) .
\end{aligned}
$$

For each complex number $z, N(z a)=|z| N(a)$. From Lemma 3.2 we conclude that, for some positive real number $r, N(a) \geqq r|a|_{0}, a \in A_{1}$. These observations show that $A_{1}$ with norm $N$ is a complex normed algebra. Since it is clear that $N(1) \leqq 1$, it follows that $N(1)=1$. This proves (4.1.1). To prove (4.1.2) suppose that \|\| is a norm on $A_{1}$ satisfying the conditions in (4.1.2). Then,

$$
\|a\| \leqq \sum\left\|a_{j}\right\| \leqq \sum\left|a_{j}\right|_{0}^{1-s}\left|a_{j}\right|_{1}^{s} \leqq N(a)+\varepsilon .
$$

This proves (4.1.2).

Consider (4.1.3). If we take $B^{k}$ to be $A_{k}$ in 2.8 and $a$ in $A_{1}=A_{0} \cap A_{1}$, we have that $M(a) \leqq|a|_{0}^{1-s}|a|_{1}^{s}$. Since by (4.1.2), $N$ is the maximal norm with this property, we can conclude (4.1.3).

Using the same argument as in Lemma 3.4, we conclude that $\phi$ is a well-defined multiplicative linear functional on $R_{x}\left(E_{1}\right)$ which is continuous with respect to the norm $N_{s}$. From Lemma 3.2 and the definition of $N$, it is clear that there is a constant $r_{1}$ such that $N(a) \leqq r_{1}|a|_{1}, a \in A_{1}$. Since $R_{x}\left(E_{1}\right)$ is dense in $A_{1}$ with respect to the norm ||$_{1}$, it is also dense with respect to the norm $N$. From these observations we can conclude that $\phi$ has a unique extension in $K_{s}$. This completes the proof of the theorem.

5. The structure spaces of the interpolated algebras. The main purpose of this section is to prove the main theorem of the paper, Theorem 5.5. This theorem gives a precise formula for the structure space of a Banach algebra obtained from the two Banach algebras in a RIS by analytic interpolation. The formula involves the structure spaces of the two given algebras and a harmonic function related to these structure spaces.

5.1 Definition. Let $E_{0}, E_{1}$ be sets in the plane and $\omega$ a function defined on $E_{1}$ such that the following conditions are satisfied: (i) $E_{0}$ is a subset of the interior of $E_{1}$ and the interior of $E_{1}$ is connected ; (ii) $\omega$ is continuous on $E_{1}$; (iii) $E_{0}$ is the zero set of $\omega$; (iv) $\omega \equiv 1$ on $\beta_{1}$, the boundary of $E_{1}$; and, (v) $\omega$ is harmonic on $U$, the complement of $E_{0}$ in the interior of $E_{1}$. When these conditions are met, we call $\left(E_{0}, E_{1}, \omega\right)$ a harmonic triple.

We note the following.

5.2 Proposition. For $0<s<1,\{z: \omega(z)<s\}$ is an open set with boundary $\{z: \omega(z)=s\}$. 
Before taking up the main theorem, we will prove the following lemma, which is, perhaps, of some interest in itself. The lemma can be regarded as a generalization of the three lines theorem (see e.g. [3, p. 520]), in which a different norm is assigned to each line $\operatorname{Re}(\xi)=s$. For notation, let $D$ denote the set of complex numbers $\xi$ with $0 \leqq \operatorname{Re}(\xi) \leqq 1$ and let $\gamma_{k}$ denote the subset $\operatorname{Re}(\xi)=k, k=0,1$. We will let $\mathscr{G}_{1}$ denote the class of functions $f(z, \xi)$ such that $f$ is continuous on $E_{1} \times D$, vanishes at infinity and is analytic on the interior of $E_{1} \times D$.

5.3 Lemma. For each $f$ in $\mathscr{G}_{1}$,

$$
\sup _{\omega(z) \leqq s}|f(z, s)| \leqq \sup _{z \in E_{0} ; t_{\text {real }}}|f(z, i t)|^{1-s} \sup _{z \in E_{1} ; t \text { real }}|f(z, 1+i t)|^{s} .
$$

Proof. Let $R$ denote the subset of $E_{1} \times D$ consisting of pairs $(z, \xi)$ such that $\omega(z)=\operatorname{Re}(\xi)(\xi=s+i t)$. Let $\omega_{1}$ be the function on $E_{1} \times D$ defined by $\omega_{1}(z, \xi)$ $=\frac{1}{2}(\omega(z)+s)$ and let $\phi=\left(1-\omega_{1}\right) \log m_{0}+\omega_{1} \log m_{1}$, where $m_{0}$ and $m_{1}$ are the maximum values that $|f|$ assumes on $E_{0} \times \gamma_{0}$ and $\beta_{1} \times \gamma_{1}$, respectively. Since the function $f / e^{\phi}$ is continuous and vanishes at infinity on the locally compact space $R,\left|f / e^{\phi}\right|$ assumes its maximum on $R$.

We will prove the following

5.4 Proposition. There is a point $\left(z_{1}, \xi_{1}\right)$ in $E_{0} \times \gamma_{0} \cup E_{1} \times \gamma_{1}$ such that

$$
|f(z, \xi) / \exp [\phi(z, \xi)]| \leqq\left|f\left(z_{1}, \xi_{1}\right) / \exp \left[\phi\left(z_{1}, \xi_{1}\right)\right]\right|
$$

for all $(z, \xi)$ in $R$.

Let us first complete the proof of the lemma using this proposition. Evidently, $\phi \equiv \log m_{0}$ on $E_{0} \times \gamma_{0}$ and $\phi \equiv \log m_{1}$ on $\beta_{1} \times \gamma_{1}$. We conclude from the proposition that $|f| e^{\phi} \mid \leqq 1$ on $R$. Therefore, for a pair $(z, \xi)$ in $R$ such that $\omega(z)=\operatorname{Re}(\xi)=s$ $(0<s<1)$ we have

$$
|f(z, \xi)| \leqq \exp [\phi(z, \xi)]=m_{0}^{1-s} m_{1}^{s} .
$$

Since $f$ is analytic on the set $\omega(z) \leqq s$ and the region $\omega(z)<s$ has boundary $\omega(z)=s$, we conclude that the last inequality above is valid for all points $(z, \xi)$ in $E_{1} \times D$ such that $\omega(z) \leqq \operatorname{Re}(\xi)$. This establishes the conclusion of the lemma. We now turn to the proof of the proposition.

To prove the proposition suppose $\left|f / e^{\phi}\right|$ does not assume its maximum over $R$ on the set $E_{0} \times \gamma_{0} \cup E_{1} \times \gamma_{1}$, and let $\left(z_{0}, \xi_{0}\right)$ be a point where the maximum is assumed. In particular, the maximum $\neq 0$. On the set $R$ we have $\omega_{1}(z, \xi)=\omega(z)$, so $\phi(z, \xi)=a+b \omega(z),(z, \xi) \in R$, for some real numbers $a$ and $b$. Let $\psi$ be the harmonic conjugate of $\omega$ at the point $z_{0}$ such that $\psi\left(z_{0}\right)=t_{0} \quad\left(\xi_{0}=s_{0}+i t_{0}\right.$ and $\xi=s+i t)$. Then the function $h=\omega+i \psi$ is analytic at $z_{0}, h\left(z_{0}\right)=\xi_{0}$ and $(z, h(z))$ is in $R$ for $z$ in some neighborhood of $z_{0}$. Since $f$ is analytic in both variables in a neighborhood of $\left(z_{0}, \xi_{0}\right)$, we conclude that the function

$$
f(z, h(z)) / \exp [a+b h(z)]
$$


is analytic in a neighborhood of $z_{0}$ and its modulus agrees with the modulus of $f / e^{\phi}$ at the points $(z, h(z))$, which lie in $R$. Therefore, the function (5.4.1) is constant and the modulus of the constant is the modulus of $f / e^{\phi}$ evaluated at $\left(z_{0}, \xi_{0}\right)$. Now let $\alpha(u)$ be a continuous complex-valued function on $0 \leqq u \leqq 1$ such that $\alpha(0)=z_{0}$, $\alpha(u) \in U$ for $0<u<1$ and $\alpha(1) \in \beta_{1}$. Let $h_{1}$ be a continuation of $h$ along the curve $\alpha$ in the direction of increasing $u$. This is possible since $\omega$ is harmonic at each point $\alpha(u), 0 \leqq u<1$. We note that $\omega(z)=\operatorname{Re} h_{1}(z)$ for $z$ in the domain of $h_{1}$, since this equation is valid in some neighborhood of $z_{0}$. Since the curve $\left(\alpha(u), h_{1}(\alpha(u))\right)$, $0 \leqq u<1$, lies in the interior of $E_{1} \times D, f$ is analytic on a neighborhood of this curve and we conclude that

$$
f\left(z, h_{1}(z)\right) / \exp \left[a+b h_{1}(z)\right]
$$

is a continuation of the function (5.4.1) along the curve $\alpha$. This means, of course, that (5.4.2) is constant. Since $f$ vanishes at infinity, $\operatorname{Im} h_{1}(z)$ is bounded, so we can choose a sequence $u_{j}, 0<u_{i}<1$, which converges to 1 and such that the sequence $\left(\alpha\left(u_{j}\right), h_{1}\left(\alpha\left(u_{j}\right)\right)\right)$ converges to some point, say $\left(z_{1}, \xi_{1}\right)$, in $\beta_{1} \times \gamma_{1}$. Since $f$ and $\phi$ are continuous on $E_{1} \times D$, we conclude from our observations that

$$
\begin{aligned}
\left|f\left(z_{1}, \xi_{1}\right) / \exp \left[\phi\left(z_{1}, \xi_{1}\right)\right]\right| & =\lim _{j}\left|f\left(\alpha\left(u_{j}\right), h_{1}\left(\alpha\left(u_{j}\right)\right)\right) / \exp \left[a+b \omega\left(\alpha\left(u_{j}\right)\right)\right]\right| \\
& =\lim _{j}\left|f\left(\alpha\left(u_{j}\right), h_{1}\left(\alpha\left(u_{j}\right)\right)\right) / \exp \left[a+b h_{1}\left(\alpha\left(u_{j}\right)\right)\right]\right| \\
& =\left|f\left(z_{0}, \xi_{0}\right) / \exp \left[\phi\left(z_{0}, \xi_{0}\right)\right]\right| .
\end{aligned}
$$

This contradiction completes the proof of the proposition.

We now turn to our main theorem.

5.5 THEOREM. Suppose that $\left(E_{0}, E_{1}, \omega\right)$ is a harmonic triple, that $E_{0}=\mathrm{sp}\left(x, A_{0}\right)$ and that $E_{1}=\operatorname{sp}\left(x, A_{1}\right)$ where $\left(A_{0}, A_{1}, W, x\right)$ is a RIS (see 3.1). If (5.5.2) implies (5.5.3) and $0<s<1$, then the following conditions on a complex number $z^{\prime}$ are equivalent:

(5.5.1) there exists $a \phi$ in $K_{s}$ such that $f\left(z^{\prime}\right)=\phi(f(x))$ for all $f$ in $R\left(E_{1}\right)$;

(5.5.2) $z^{\prime}$ is in $E_{1}$ and

for all $f$ in $R\left(E_{1}\right)$;

$$
\left|f\left(z^{\prime}\right)\right| \leqq \sup _{z \in E_{0}}|f(z)|^{1-s} \sup _{z \in E_{1}}|f(z)|^{s}
$$

(5.5.3) $\omega\left(z^{\prime}\right) \leqq s$;

(5.5.4) $z^{\prime}$ is in $E_{1}$ and

for all $q$ in $\mathscr{G}_{1}$

$$
\left|q\left(z^{\prime}, s\right)\right| \leqq \max _{k=0,1} \sup _{z \in E_{k} ; t \text { real }}|q(z, k+i t)|
$$

(5.5.5) there exists $a \phi$ in $H_{s}$ such that $f\left(z^{\prime}\right)=\phi(f(x))$ for all $f$ in $R\left(E_{1}\right)$.

5.6 COMment. We conjecture that with the hypothesis of Theorem 5.5, (5.5.2) implies (5.5.3); however, we have not been able to prove this. But, we can deduce (5.5.3) from (5.5.2) if we further restrict $E_{0}$ and $E_{1}$. This is the purpose of our next lemma. 
5.7 Lemma. Suppose that $E_{0}$ and $E_{1}$ are compact sets such that there exist an analytic function $F$ on a connected open set $V$ and positive real numbers $r_{0}$ and $r_{1}$ $\left(r_{0}<r_{1}\right)$ such that $E_{k}=\left\{z \in V:|F(z)| \leqq r_{k}\right\}, k=0,1$. Let $\omega(z)=0$ for $z$ in $E_{0}$ and

$$
\omega(z)=\frac{\log |F(z)|-\log r_{0}}{\log r_{1}-\log r_{0}}, \quad z \in E_{1} \mid E_{0} .
$$

Then $\left(E_{0}, E_{1}, \omega\right)$ is a harmonic triple and (5.5.2) implies (5.5.3).

Proof. The fact that $\left(E_{0}, E_{1}, \omega\right)$ is a harmonic triple is clear. From (5.5.2) and Runge's theorem we see that $\left|F\left(z^{\prime}\right)\right| \leqq r_{0}^{1-s} r_{1}^{s}$; and from this we conclude that $\omega\left(z^{\prime}\right) \leqq s$.

Proof of Theorem 5.5. If $z^{\prime}$ satisfies (5.5.1), then for each $f$ in $R\left(E_{1}\right)$ we have

$$
\left|f\left(z^{\prime}\right)\right|=\left|f^{n}\left(z^{\prime}\right)\right|^{1 / n}=\left|\phi\left(f^{n}(x)\right)\right|^{1 / n} \leqq N\left(f^{n}(x)\right)^{1 / n} .
$$

From (4.1.2) we know that the last term does not exceed

$$
\left(\left|f^{n}(x)\right|_{0}^{1-s}\left|f^{n}(x)\right|_{1}^{s}\right)^{1 / n}
$$

This last expression, as a consequence of the spectral radius formula for Banach algebras, converges to

$$
\rho\left(f(x), A_{0}\right)^{1-s} \rho\left(f(x), A_{1}\right)^{s}
$$

where $\rho\left(f(x), A_{k}\right)$ denotes the spectral radius of $f(x)$ as an element in $A_{k}$. From the spectral mapping theorem we have that

$$
\rho\left(f(x), A_{k}\right)=\sup _{z \in E_{k}}|f(z)|, \quad k=0,1 .
$$

Our observations clearly yield (5.5.2).

That (5.5.2) implies (5.5.3) is part of our hypothesis. It follows from Lemma 5.3 that (5.5.3) implies (5.5.4).

To prove that (5.5.4) implies (5.5.5) we suppose that $z^{\prime}$ satisfies (5.5.4). For each $z$ in $E_{1}$ let $\lambda(z, \cdot)$ denote the element of the structure space of $A_{1}$ as described in Lemma 3.4. So, $\lambda(z, f(x))=f(z), f \in R\left(E_{1}\right)$, and the function $\lambda(\cdot, y)$, for each $y$ in $A_{1}$, is continuous on $E_{1}$ and analytic on the interior of $E_{1}$. Let $y$ be an element in $A_{1}$ and $\varepsilon$ a positive real number. By taking $\left(A_{0}, A_{1}\right)$ as the interpolation pair in Lemma 2.5 and noting that $A_{1}=A_{0} \cap A_{1}$, we can choose $q$ in $\mathscr{G}, q(\xi)=\sum c_{j}(\xi) y_{j}$ $(j=1,2, \ldots, N)$, so that $q(s)=y$ and $\|q\|_{\mathscr{F}} \leqq M_{s}(y)+\varepsilon$. The function $\lambda(z, q(\xi))$ $=\sum c_{j}(\xi) \lambda\left(z, y_{j}\right)$ is in the class $\mathscr{G}_{1}$. From (5.5.4), Lemma 3.5 and the above observations we obtain the following inequalities.

$$
\begin{aligned}
\left|\lambda\left(z^{\prime}, y\right)\right|=\left|\lambda\left(z^{\prime}, q(s)\right)\right| & \leqq \max _{k=0,1} \sup _{t \text { real }} \sup _{z \in E_{k}}|\lambda(z, q(k+i t))| \\
& \leqq \max _{k=0,1} \sup _{t \text { real }}|q(k+i t)|_{k}=\|q\|_{\mathscr{F}} \leqq M_{s}(y)+\varepsilon .
\end{aligned}
$$

From this we see that $\lambda\left(z^{\prime}, \cdot\right)$ is a nonzero multiplicative linear functional on $A_{1}$ which is bounded with respect to the norm $M_{s}$. From Lemma 2.2 we know that $A_{1}$ 
is dense in $A_{s}$, so $\lambda\left(z^{\prime}, \cdot\right)$ has a unique extension $\phi$ in $H_{s}$. For each $f$ in $R\left(E_{1}\right)$, $\phi(f(x))=\lambda\left(z^{\prime}, f(x)\right)=f\left(z^{\prime}\right)$. This establishes (5.5.5).

Finally, we will show that (5.5.5) implies (5.5.1). Let $\phi$ correspond to $z^{\prime}$ as in (5.5.5). From the fact that $\phi(f(x))$ is in $\operatorname{sp}\left(f(x), A_{s}\right)$ and (4.1.3), we conclude that the inequalities

$$
\left|f\left(z^{\prime}\right)\right|=|\phi(f(x))| \leqq M_{s}(f(x)) \leqq N_{s}(f(x))
$$

are valid for all $f$ in $R\left(E_{1}\right)$. We conclude from (4.1.4) that there is an element $\phi_{1}$ in $K_{s}$ such that $f\left(z^{\prime}\right)=\phi_{1}(f(x)), f \in R\left(E_{1}\right)$. This completes the proof of Theorem 5.5.

6. The spectrum of an operator on an interpolation space. The purpose of this section is to obtain an upper bound for the spectrum of an operator on an interpolation space in terms of its spectra relative to the two given spaces. Theorem 6.7 is the main result. Corollaries 6.8 and 6.9 combine 6.7 and 5.5 to get precise formulas for the upper bounds. Theorem 6.10 is a refinement of Theorem 6.7. For notation, if $T$ is a linear transformation on a complex vector space $X$ which is bounded with respect to a norm \|\| on $X$, then we let sp $(T, X,\|\|)$ denote the spectrum of $T$ as an element of the Banach algebra of bounded operators on $X$ with respect to the norm \|\| .

6.1 Definition. Let $\left(B^{0}, B^{1}\right)$ be an interpolation pair with associated $V$ as defined in 2.1 and assume that $B^{0} \cap B^{1}$ is dense in $B^{0}$ and $B^{1}$. Let $T$ be a linear transformation on $B^{0} \cap B^{1}$ which is bounded with respect to the norms of $B^{0}$ and $B^{1}$ restricted to $B^{0} \cap B^{1}$. For convenience, we let $E_{k}$ denote $\operatorname{sp}\left(T, B^{0} \cap B^{1},\|\|_{k}\right)$ we assume that

(6.1.1) one of the sets $E_{0}$ and $E_{1}$ is contained in the interior of the other. When these conditions are met we say that $T$ is an interpolation operator with respect to the interpolation pair $\left(B^{0}, B^{1}\right)$.

For normed linear spaces $B$ and $C$ we let $O(B, C)$ denote the Banach space of bounded linear transformations from $B$ into $C$, and we use $O(B)$ in place of $O(B, B)$. The norm in each case is the operator norm.

6.2 Definition. Let $A_{k}$ be the commutative Banach algebra obtained by taking the closure of the algebra $\left\{f(T): f \in R\left(E_{k}\right)\right\}$ in $O\left(B_{k}\right)$. Let $W=O\left(B^{0} \cap B^{1}, B^{0}+B^{1}\right)$ with the uniform topology. Each operator $U$ in $A_{k}$ clearly determines an element $U \sim$ in $W$, where $U^{\sim}$ is the restriction of $U$ to $B^{0} \cap B^{1}$; furthermore, since $B^{0} \cap B^{1}$ is dense in $B^{k}$, the map $U \rightarrow U^{\sim}$ is clearly continuous and 1-1. Thus, we can consider $A_{0}$ and $A_{1}$ as continuously embedded in $W$. From Proposition 6.3 we know that either $\left(A_{0}, A_{1}, W, T\right)$ or $\left(A_{1}, A_{0}, W, T\right)$ is a RIS, and since $\left[A_{0}, A_{1}\right]_{s}$ $=\left[A_{1}, A_{0}\right]_{1-s}$ it follows from Lemma 3.3 that $A_{s}=\left[A_{0}, A_{1}\right]_{s}$ is a commutative Banach algebra. We call the structure space $H_{s}$ of $A_{s}$ the structure space associated with $T$.

6.3 Proposition. If $E_{0}$ is contained in the interior of $E_{1}$, then $\left(A_{0}, A_{1}, W, T\right)$ is a RIS. If $E_{1}$ is contained in the interior of $E_{0}$, then $\left(A_{1}, A_{0}, W, T\right)$ is a RIS. 
The proof is an easy consequence of the definitions of $A_{0}$ and $A_{1}$ and the obvious fact that $E_{k}=\operatorname{sp}\left(T, A_{k}\right), k=0,1$.

6.4 CoMmeNT. In the definition of a RIS it is assumed that $\operatorname{sp}\left(x, A_{0}\right)$ is contained in the interior of $\operatorname{sp}\left(x, A_{1}\right)$. This apparent asymmetry in the definition is usually not troublesome; for if the spectrum of $x$ with respect to one of the algebras $A_{0}$ and $A_{1}$ is contained in the interior of the spectrum of the other, then we can take the first algebra in $\left(A_{0}, A_{1}, V, x\right)$ to correspond to the smaller spectrum. We allowed this apparent asymmetry (actually, by using some more notation and conventions, this asymmetric condition could be eliminated from the definitions of a RIS without affecting the results) for notational convenience. However, it turns out that in Theorem 6.10 and $\S 7$ we cannot choose notation so that the first algebra in $\left(A_{0}, A_{1}, W, T\right)$ has the smaller associated spectrum, so we have not insisted in Definition 6.1 that $E_{0}$ is contained in the interior of $E_{1}$. This causes only a slight inconvenience when we apply the results about RIS's in the next lemmas.

Throughout this section $T$ will denote an operator satisfying 6.1. We will use the notation in Definitions 6.1 and 6.2 to denote the various objects associated with $T$ in these definitions. We let \|\|$_{s}$ and $M_{s}$ denote the norms of the spaces $B_{s}=\left[B^{0}, B^{1}\right]_{s}$ and $A_{s}=\left[A_{0}, A_{1}\right]_{s}$, respectively. For each $a$ in $A_{0} \cap A_{1}$, let $J_{s}(a)$ denote the operator norm of $a$ with respect to the norm \|\|$_{s}$ restricted to $B^{0} \cap B^{1}$.

6.5 LEMMA. $T$ is bounded with respect to the norm \|\|$_{s}$ on $B^{0} \cap B^{1}$ and

$$
\operatorname{sp}\left(T, B^{0} \cap B^{1},\|\|_{s}\right) \subset E_{0} \cup E_{1} .
$$

Proof. The first assertion follows from [2, 4, p. 115]. If $\lambda$ is in the complement of $E_{0} \cup E_{1}$, then the operator $\lambda I-T$ ( $I$ being the identity operator on $\left.B^{0} \cap B^{1}\right)$ has an inverse which is a bounded operator with respect to the norms \|\|$_{0}$ and \|\|$_{1}$ on $B^{0} \cap B^{1}$; thus, from [2, 4, p. 115] we conclude that this inverse is also bounded with respect to the norm \|\|$_{s}$, on $B^{0} \cap B^{1}$. This shows that $\lambda$ is not in

$$
\operatorname{sp}\left(T, B^{0} \cap B^{1},\|\|_{s}\right),
$$

which completes the proof.

6.6 LeMmA. $J_{s} \leqq M_{s}$ on $A_{0} \cap A_{1}$.

Proof. Let $a$ be in $A_{0} \cap A_{1}$. Choose $x$ in $B^{0} \cap B^{1}$ so that $\|x\|_{s}=1$ and $J_{s}(a)$ $\leqq\|a x\|_{s}+\varepsilon$, where $\varepsilon$ is a positive real number. From the definition of \|\|$_{s}$ we can choose $q_{1}$ in $\mathscr{F}\left(B^{0}, B^{1}\right)$ so that

$$
q_{1}(s)=x \quad \text { and } \quad\left\|q_{1}\right\|_{F_{\left(B^{0}, B^{1}\right)}} \leqq\|x\|_{s}+\varepsilon .
$$

By Lemma 2.5 we can choose $q$ in $\mathscr{G}\left(A_{0}, A_{1}\right)$ so that

$$
q(s)=a \quad \text { and } \quad\|q\|_{\mathscr{F}_{\left(A_{0}, A_{1}\right)}} \leqq M_{s}(a)+\varepsilon .
$$


By definition of $\mathscr{G}, q$ has the form $q(\xi)=\sum c_{j}(\xi) a_{j}(j=1, \ldots, N)$, where, in particular, each $a_{j}$ is in $A_{0} \cap A_{1}$. An easy computation shows that each element of $A_{0} \cap A_{1}$ is bounded with respect to the norm of $B^{0}+B^{1}$ restricted to $B^{0} \cap B^{1}$. We conclude from our observations that $q(\xi) q_{1}(\xi)$ is a function in $\mathscr{F}\left(B^{0}, B^{1}\right)$. Furthermore,

$$
\|a x\|_{s} \leqq\left\|q q_{1}\right\|_{\mathscr{F}_{\left(B^{0}, B^{1}\right)}} \leqq\|q\|_{\left.\mathscr{F}_{\left(A_{0}, A_{1}\right.}\right)}\left\|q_{1}\right\|_{\mathscr{F}_{\left(B^{0}, B^{1}\right)} \leqq} \leqq\left(M_{s}(a)+\varepsilon\right)(1+\varepsilon) .
$$

This completes the proof.

A simple argument shows that any nonzero multiplicative linear functional on $R(E)$ is of the form $f \rightarrow f(z)$ for some $z$ in $E$. From Lemma 3.2 we know that $R_{x}\left(E_{1}\right)$ is dense in $A_{s}$ with respect to the norm $M_{s}$. From these two observations it is clear that $H_{s}$ is put in 1-1 correspondence with a subset of complex numbers by the map $\phi \rightarrow z$ where $\phi(f(x))=f(z), f \in R\left(E_{1}\right)$. We will assume this identification in the remainder of this section without further discussion.

6.7 TheOREM. $\mathrm{sp}\left(T, B^{0} \cap B^{1},\|\quad\|_{s}\right) \subset H_{s}$.

Proof. As in the proof of Lemma 6.6 we may assume that $E_{0}$ is contained in the interior of $E_{1}$, for if $A_{0}, A_{1}$ and $B_{0}, B_{1}$ are interchanged, then \|\|$_{s}$ is replaced by \|\|$_{1-s}$ and $H_{s}$ is replaced by $H_{1-s}$, so the conclusion remains valid. From our assumption we have that $\left(A_{0}, A_{1}, W, T\right)$ is a RIS.

Suppose that $z$ is in $\operatorname{sp}\left(T, B^{0} \cap B^{1},\|\|_{s}\right)$ and that $f$ is in $R\left(E_{0} \cup E_{1}\right)$. By Lemma $6.5 z$ is in $E_{0} \cup E_{1}$. Since $f$ is analytic on $\operatorname{sp}\left(T, B^{0} \cap B^{1},\|\|_{s}\right)$ it follows from the spectral mapping theorem [3, p. 569] that $f(z) \in \operatorname{sp}\left(f(T), B^{0} \cap B^{1},\|\|_{s}\right)$. Therefore, $|f(z)| \leqq J_{s}(f(T))$. Each such $f(T)$ is in $A_{0} \cap A_{1}$, so combining this last inequality with Lemma 6.6 we have

$$
|f(z)| \leqq M_{s}(f(T)), \quad f \in R\left(E_{0} \cup E_{1}\right) .
$$

As we have already seen, $\left(A_{0}, A_{1}, W, T\right)$ is a RIS, so it follows from (6.5.1) and Lemma 3.4 that $z \in H_{s}$, which completes the proof.

The following corollaries are immediate consequences of Theorem 5.5 and Theorem 6.7.

6.8 COROLlary. If, in addition to the assumptions of Theorem 6.7, there is a function $\omega$ such that $\left(E_{0}, E_{1}, \omega\right)$ is a harmonic triple and $(5.5 .2) \Rightarrow(5.5 .3)$, then

$$
\operatorname{sp}\left(T, B^{0} \cap B^{1},\|\|_{s}\right) \subset\left\{z \in E_{1}: \omega(z) \leqq s\right\} .
$$

6.9 Corollary. If, in addition to the assumptions of Theorem 6.7, the sets $E_{0}$ and $E_{1}$ are determined by a function $F$ as in Lemma 5.7, then

$$
\operatorname{sp}\left(T, B^{0} \cap B^{1},\|\|_{s}\right) \subset\left\{z \in V:|F(z)| \leqq r_{0}^{1-s} r_{1}^{s}\right\} .
$$

We will now use Theorem 6.7 to obtain a stronger theorem of the same type. For each $T$-invariant subspace $X$ of $B^{0} \cap B^{1}$, let $E_{k}(X)$ denote the set

$$
\operatorname{sp}\left(T \mid X, X,\|\|_{k}\right) \text {. }
$$


If $X^{k}$ denotes the closure of $X$ in $B^{k}(k=0,1)$, then since $X^{0}$ and $X^{1}$ are continuously embedded in $V,\left(X^{0}, X^{1}\right)$ is an interpolation pair. There is no loss in generality in assuming that $X=X^{0} \cap X^{1}$. If we further assume that one of the sets $E_{0}(X)$, $E_{1}(X)$ is contained in the interior of the other, then $T \mid X$ is an interpolation operator with respect to the interpolation pair $\left(X^{0}, X^{1}\right)$. Under these conditions we let $H_{s}(X)$ denote the structure space associated with $T \mid X$ as in (6.2).

6.10 THEOREM. Suppose that $B^{0} \cap B^{1}$ is the direct sum of T-invariant subspaces $X_{j}(j=1,2, \ldots, n)$ such that for each $j$ the projection $P_{j}$ of $B^{0} \cap B^{1}$ onto $X_{j}$ satisfies the conditions $\left\|P_{j} x\right\|_{k} \leqq c\|x\|_{k}, k=0,1 ; x \in B^{0} \cap B^{1}$ ( $c$ is a constant). If for each $j$ one of the sets $E_{0}\left(X_{j}\right)$ and $E_{1}\left(X_{j}\right)$ is contained in the interior of the other, then

$$
\operatorname{sp}\left(T, B^{0} \cap B^{1},\|\|_{s}\right) \subset \bigcup_{j=1,2, \ldots, n} H_{s}\left(X_{j}\right) .
$$

Proof. From the fact that each $P_{j}$ is bounded with respect to the norms \|\|$_{0}$, \|\|$_{1}$ one can easily see that $P_{j}\left(\left[B^{0}, B^{1}\right]_{s}\right)=\left[X_{j}^{0}, X_{j}^{1}\right]_{s}$ and that the norm \|\|$_{s}$ restricted to $P_{f}\left(\left[B^{0}, B^{1}\right]_{s}\right)$ is equivalent to the norm of $\left[X_{j}^{0}, X_{j}^{1}\right]_{s}$. We conclude from Theorem 6.7 that

$$
\operatorname{sp}\left(T \mid X_{j}, X_{j},\|\|_{s}\right) \subset H_{s}\left(X_{j}\right), \quad j=1,2, \ldots, n .
$$

From the assumptions on $P_{f}$ and $\left[2,4\right.$, p. 115] we conclude that $P_{j}$ is a bounded operator with respect to the norm \|\|$_{s}$ on $B^{0} \cap B^{1}$; consequently, one easily sees that $\operatorname{sp}\left(T, B^{0} \cap B^{1},\|\|_{s}\right)$ is the union (over $\left.j\right)$ of the sets which appear on the left-hand side of (6.10.1). This completes the proof.

7. An example related to the main theorem. The purpose of this section is to show that containment in Theorem 6.7 may be proper. Our result is 7.3. We first introduce some notation and prove a lemma. For each positive real number $r$ and for $1<p<\infty$, let $H(r, p)$ denote the completion of the complex polynomials $f$ with respect to the norm

$$
\left(\int_{0}^{2 \pi}\left|f\left(r e^{i \theta}\right)\right|^{p} d \theta\right)^{1 / p}
$$

For convenience, we let $r_{0}, r_{1}$ be two positive real numbers and $p_{0}, p_{1}$ numbers with $1<p_{0}, p_{1}<\infty$. If we take $V$ to be $H(u, 1)$, where $u$ is a positive real number $\leqq$ both $r_{0}$ and $r_{1},\left(H\left(r_{0}, p_{0}\right), H\left(r_{1}, p_{1}\right)\right)$ is an interpolation pair. Let

$$
C_{s}=\left[H\left(r_{0}, p_{0}\right), H\left(r_{1}, p_{1}\right)\right]_{s}, \quad B_{s}=\left[H\left(1, p_{0}\right), H\left(1, p_{1}\right)\right]_{s} .
$$

7.1 Lemma. For $0<s<1$ we have $C_{s}=H(r, p)$, where $1 / p=(1-s) / p_{0}+s / p_{1}$ and $r=r_{0}^{1-s} r_{1}^{s}$.

Proof. For each polynomial $f$ and each complex number $\xi=s+i t, 0 \leqq s \leqq 1$, we let $T_{\xi} f$ denote the polynomial with value $f\left(r_{0}^{\xi-1} r_{1}^{-\xi} z\right)$ at $z$, where $z$ is an arbitrary complex number. We need the following proposition.

7.2 Proposition. For each polynomial $f,\left\|T_{s} f\right\|_{C_{s}}=\|f\|_{B_{s}}$. 
We first prove the lemma and then the proposition. If we let $L_{p}$ denote the class of functions $f\left(e^{i \theta}\right)$ such that $\int_{0}^{2 \pi}\left|f\left(e^{i \theta}\right)\right|^{p} d \theta<\infty$, then it follows from the wellknown theorem of M. Riesz (e.g., see $[8$, p. 151]) that $H(1, p)$ has a complementary subspace in $L_{p}, 1<p<\infty$. From this and the elementary fact that analytic interpolation distributes over direct sums in the Banach space sense, we see that $H(1, p)=B_{s}$ and the norms are equivalent. From this and the proposition we have that for each polynomial $f$,

$$
\|f\|_{C_{s}}=\left\|T_{s}^{-1} f\right\|_{B_{s}} \sim\left\|T_{s}^{-1} f\right\|_{H(1, p)}=\|f\|_{H(r, p)}
$$

where $a \sim b$ means that $a / b$ is between two fixed positive real numbers. One of the spaces $H\left(r_{0}, p_{0}\right), H\left(r_{1}, p_{1}\right)$, call it $D$, is contained in the other. Let $E$ denote the other space. It is easy to see that for some constant $k,\|\|_{E} \leqq k\|\|_{D}$ on $D$. From this and the definition of analytic interpolation it is clear that \|\|$_{C_{s}} \leqq k\|\|_{D}$ on $D$. Since the polynomials are dense in $D$ with respect to \|\|$_{D}$ and $D$ is dense in $C_{s}$ with respect to \|\|$_{C_{s}}$ by Lemma 2.3, we see that the polynomials are dense in $C_{s}$ with respect to \|\|$_{C_{s}}$. The polynomials are also dense in $H(r, p)$. From these observations and (7.2.1) we conclude that $C_{s}=H(r, p)$.

To prove the proposition let $f$ be a polynomial and $\varepsilon$ a positive real number. We first show that there is a $q(\xi)(\xi=s+i t), 0 \leqq s \leqq 1$, of the form $q(\xi)=\sum c_{n}(\xi) f_{n}$ such that: each $c_{n}$ is continuous on $0 \leqq s \leqq 1$, analytic on the interior, vanishes at infinity; each $f_{n}$ is a polynomial; $q(s)=f$; and $\|f\|_{C_{s}}+\varepsilon \geqq\|q\|_{\mathscr{F}}$, where

$$
\mathscr{F}=\mathscr{F}\left(H\left(r_{0}, p_{0}\right), H\left(r_{1}, p_{1}\right)\right) \text {. }
$$

To see this first choose $q_{1}$ in $\mathscr{G}$ so that $q_{1}(s)=f$ and $\|f\|_{C_{s}}+\varepsilon / 2 \geqq\left\|q_{1}\right\| \mathscr{F}$. By definition of $\mathscr{G}, q_{1}(\xi)=\sum c_{n}(\xi) x_{n}(n=1, \ldots, N)$, where, in particular, each $x_{n}$ is in $D$. Since the polynomials are dense in $D$ we can choose polynomials $f_{n}$ so that

$$
\sum\left|c_{n}(\xi)\right|\left|x_{n}-f_{n}\right|_{D}<\varepsilon / 4 k, \quad 0 \leqq s \leqq 1 .
$$

Let $g$ denote the polynomial $\sum c_{n}(s)\left(x_{n}-f_{n}\right)$. For sufficiently small $\eta$ it is clear that the function

$$
q(\xi)=\sum c_{n}(\xi) f_{n}+e^{\eta(\xi-s)^{2}} g
$$

has the desired properties.

Taking $f$ and $q$ as above the following inequalities are evident:

$$
\begin{aligned}
\|f\|_{C_{s}}+\varepsilon \geqq\|q\|_{\mathscr{F}} & =\max _{k=0,1} \sup _{t \text { real }}\|q(k+i t)\|_{H\left(r_{k}, p_{k}\right)} \\
& \geqq \max _{k=0,1} \sup _{t \text { real }}\left\|T_{k+i t}^{-1} q(k+i t)\right\|_{H\left(1, p_{k}\right)} \geqq\left\|T_{s}^{-1} f\right\|_{B_{s}} .
\end{aligned}
$$

We have shown that for each polynomial $f,\left\|T_{s} f\right\|_{C_{s}} \geqq\|f\|_{B_{s}}$. The opposite inequality can be proved in an analogous manner, which completes the proof of the proposition. 
7.3 Example. Our purpose is to show that the sets in Theorem 6.7 need not be equal. Let $p$ be a fixed real number, $1<p<\infty$, and let $H(r, p)=H(r)$ for convenience. Let $B^{0}$ be $H(1 / 4) \oplus H(1 / 9)$ and $B^{1}$ be $H(1 / 9) \oplus H(1)$. We take $B^{0}$ and $B^{1}$ to be embedded in $H(1 / 9,1) \oplus H(1 / 9,1)$ in the natural way. The operator $T$ is defined on $B^{0}$ and $B^{1}$ as multiplication in each coordinate by the function $f(z)=z$. Evidently, the spectrum of $T$ as an operator on $B^{0}$ is $|z| \leqq 1 / 4$ and the spectrum of $T$ as an operator on $B^{1}$ is $|z| \leqq 1$. It follows from Lemma 5.7 that $H_{1 / 2}=\{z:|z| \leqq 1 / 2\}$. On the other hand, from the distribution of analytic interpolation over direct sums and Lemma 7.1 we conclude that

$$
\left[B^{0}, B^{1}\right]_{1 / 2}=[H(1 / 4), H(1 / 9)]_{1 / 2} \oplus[H(1 / 9), H(1)]_{1 / 2}=H(1 / 6) \oplus H(1 / 3) .
$$

Thus, $\operatorname{sp}\left(T, B^{0} \cap B^{1},\|\|_{1 / 2}\right)$ is the set $\{z:|z| \leqq 1 / 3\}$, which is properly contained in $H_{1 / 2}$.

\section{BiBLIOGRAPHY}

1. Errett Bishop, Holomorphic completions, analytic continuation, and the interpolation of semi-norms, Ann. of Math. 78 (1963), 468-500.

2. A. P. Calderón, Intermediate spaces and interpolation, the complex method, Studia Math. 24 (1964), 113-190.

3. Nelson Dunford and Jacob T. Schwartz, Linear operators, Part I, Interscience, New York, 1958.

4. C. J. A. Halberg, Jr., The spectra of bounded linear operators on the sequence spaces, Proc. Amer. Math. Soc. 8 (1957), 728-732.

5. - Semigroups of matrices defining linked operators with different spectra, Pacific J. Math. 13 (1963), 1187-1191.

6. C. J. A. Halberg, Jr. and Angus E. Taylor, On the spectrum of linked operators, Pacific J. Math. 6 (1956), 283-290.

7. E. Hille and R. S. Phillips, Functional analysis and semigroups, Amer. Math. Soc. Colloq. Publ. Vol. 31, Amer. Math. Soc., Providence, R. I., 1957.

8. Kenneth Hoffman, Banach spaces of analytic functions, Prentice-Hall, Englewood Cliffs, N. J., 1962.

9. J. L. Lions, Une construction d'spaces d'interpolation, C. R. Acad. Sci. Paris 83 (1960), 1853-1855.

10. E. C. Titchmarsh, The theory of functions, 2nd ed., Oxford Univ. Press, London, 1939.

University of CALIFornia, Riverside, CALIForNia 